\title{
Evolution de la nappe phréatique depuis un siècle dans Paris et niveaux connus en 1990
}

\author{
Evolution of the phreatic water table for a century in Paris \\ and known nevels on 1990
}

\author{
A.M. PRUNIER-LEPARMENTIER \\ Inspection générale des carrières*
}

Rev. Franç. Géotech. n 56, pp. 67-75 (juillet 1991)

\section{Résumé}

A une impressionnante baisse des niveaux à Paris, essentiellement en rive droite, qui connut son maximum vers 1970-71, succéda une remontée rapide mais de ce fait moins importante.

Depuis 1983 environ les piézomètres suivis par l'Inspection générale des Carrières semblent indiquer un nouvel état d'équilibre. Dans quelle mesure ces deux années de sécheresse 89 et 90 ne vont-elles pas le modifier?

\section{Abstract}

After a sever drowdown of the water table in Paris, mainly on the right bank, the maximum of which during 1970-71, a fast uproard movement occurred but not so large.

Since about 1983 the piezometers which are surveyed by the "Inspection générale des Carrières" seem to show a new equilibrium. Are modifications to appear after the two very dry years 1989-1990?

\footnotetext{
- 1, place Denfert-Rochereau, 75014 Paris.
} 
La nappe phréatique, ou première nappe rencontrée dans le sous-sol, suscite un intérêt particulier depuis une vingtaine d'années, et les mesures de son niveau se sont intensifiées, en raison des fortes remontées enregistrées dans certains secteurs de la capitale.

Des synthèses sur l'évolution de la nappe phréatique depuis 1858 ont été réalisées: DELESSE (1858), DIFFRE (1965), MARVY (1985), COLIN (1986), PRUNIER-LEPARMENTIER (1988). Parallèlement, l'Inspection générale des Carrières suit régulièrement un réseau de piézomètres répartis dans Paris.

Trois périodes doivent être distinguées. La première représente la baisse générale du niveau des nappes, elle dure un siècle environ et s'arrête en 1970-71. La deuxième intègre toute la remontée et dure environ 12-13 ans. Enfin la troisième est la période actuelle où semble-t-il un nouvelle équilibre s'instaure. Elle caractérise les 6 dernières années.

\section{LE CONCEPT DE NAPPE PHRÉATIQUE A PARIS}

Géologiquement, on peut définir quatre grands ensembles dans Paris (fig. 1) :

- le premier est constitué par la plaine alluviale. C'est le domaine de la nappe alluviale;

- le deuxième au sud est établi sur la plate-forme lutétienne. Le calcaire grossier est fortement exploité dans les zones hors d'eau pour les carrières. La nappe phréatique est lutétienne et se situe préférentiellement dans le calcaire grossier moyen voire inférieur, généralement sous le pied de carrière;

- le troisième est symbolisé par la plate-forme du marno-calcaire de Saint-Ouen. C'est le domaine de la nappe dite de l'Eocène supérieur;

- la quatrième zone se superpose à la troisième au niveau des buttes. Sur le sommet, on rencontre les nappes perchées dites des glaises vertes. Sur les flancs, les formations de pente amènent des circulations d'eau qu'il est difficile à contrôler.

Les 3 premiers ensembles définissent chacun leur propre nappe phréatique. Il existe donc à Paris 3 nappes formant la nappe phréatique. La nappe alluviale présente toutefois une particularité : elle est souvent « soutenue " par la nappe comprise dans l'aquifère sous-jacent. Par exemple, dans le sud du $15^{e}$ arrondissement, la nappe de la craie a sensiblement le même niveau piézométrique que celle des alluvions. Au nord du $15^{\mathrm{e}}$ la nappe alluviale s'écoule sur l'argile plastique et connaît un début de dépression. Dans le centre de Paris, les alluvions anciennes sont souvent sèches, la nappe phréatique était constituée par la nappe lutétienne.

\section{EVOLUTION DE LA NAPPE PHRÉATIQUE JUSQU'AUX ANNÉES 1970}

Essentiellement deux cartes hydrogéologiques résument cette évolution pendant un siècle. La carte de DELESSE (fig. 2) donne un aperçu des niveaux de



Fig. 1. - Coupe géologique schématique de Paris avec un ordre de grandeur des coefficients de perméabilité des différents horizons en $\mathrm{m} / \mathrm{s}$ ) (tirée de PRUNIER-LEPARMENTIER, 1984).

Fig. 1. - Schematic geological cut through Paris with the order of magnitude of the different layers permeability $(\mathrm{m} / \mathrm{s})$. From PRUNIER-LEPARMENTIER, 1984 


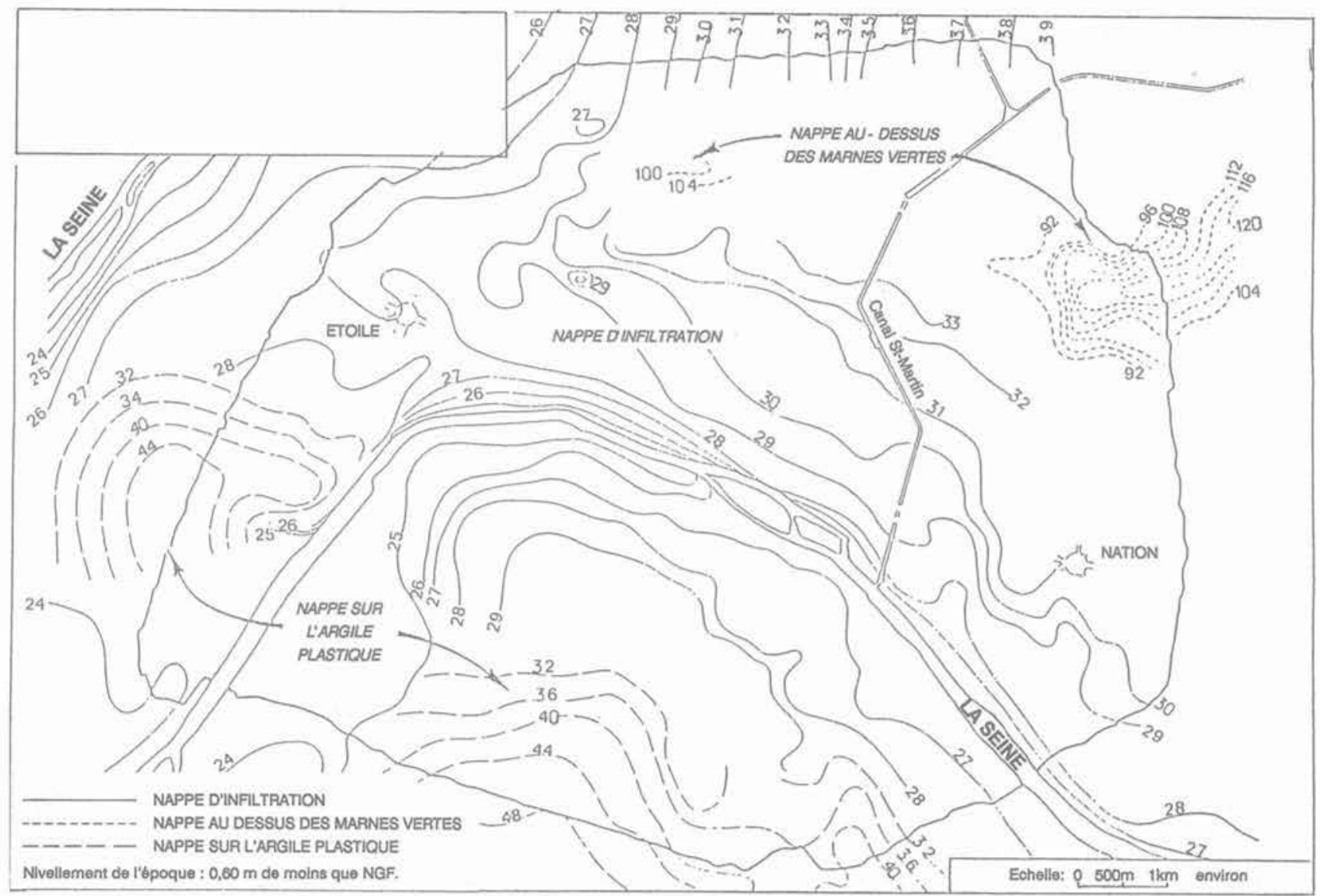

Fig. 2. - Piézométrie en 1858. Extrait de la carte hydrologique du département de la Seine (par DELESSE). Fig. 2. - 1858 piezometric map. From DELESSE.

nappes au siècle dernier. Elle sert actuellement de référence pour le niveau des nappes superficielles. A cette époque, les pompages étaient relativement faibles et les infiltrations directes d'eau pluviale ou autre dans le sol. Ce dernier n'était pas encore étanchéifié par l'urbanisation.

En comparant cette carte à celle de DIFFRE en 1965 (fig. 3), on constate qu'en rive droite les tracés se sont fortement modifiés, les niveaux piézométriques sont très nettement inférieurs à la cote $+20,00$ NGF (système orthométrique ou Lallemand) pour Paris centre rive droite. Dans ce secteur, la Seine alimente la nappe. Les travaux du RER aux Halles ont montré que les alluvions anciennes étaient sèches.

On peut également observer une baisse sensible dans le quartier de l'Etoile. Bien que moins accentuée, cette chute des niveaux est évaluée à $8 \mathrm{~m}$ par endroit (actuellement $4 \mathrm{~m}$ ).

En rive gauche le comportement de la nappe phréatique semble inchangé.

Cette baisse est la conséquence de cinq phénomènes : - le premier, plus évident, est l'installation humaine sur le site de Paris. Les eaux souterraines et de Seine ont été toutefois interdites à la consommation courante et ont été essentiellement utilisées pour les usages industriels;
- les pompages industriels se sont intensifiés, surtout dans la nappe yprésienne autour d'Aubervilliers. Mais leur influence est déjà très sensible en centre rive droite. Actuellement il est encore difficile d'évaluer le total et la répartition dans le détail des débits prélevés ;

- les différents grands travaux de ces 15 dernières années constituent la troisième cause. Par exemple pour le centre PTT Marat, $150 \mathrm{~m}^{3} / \mathrm{h}$ ont été pompés pendant un an, de décembre 1974 à février 1976. On peut citer également le Forum des Halles avec $60 \mathrm{~m}^{3} / \mathrm{h}$, plusieurs pompages de 20 à $100 \mathrm{~m}^{3} / \mathrm{h}$ dans les galeries du RER lors de sa construction...

Pour les années de 1965 à 1977 le débit moyen total, dû aux différents chantiers temporaires dans Paris intramuros, a été estimé à $500 \mathrm{~m}^{3} / \mathrm{h}$ soit environ 5 millions de $\mathrm{m}^{3} /$ an en moyenne avec un maximum en 1971 et 7 millions de $\mathrm{m}^{3}$ et un minimum de 2 millions de $\mathrm{m}^{3}$ en 1977 (BRGM 79 SGN 086 IDF) ; - Le drainage permanent dans les ouvrages souterrains existants constitue le quatrième facteur. Le métro, les canalisations et les égouts forment autant de galeries drainantes qui font plafonner le niveau de la nappe phréatique ou qui facilitent les circulations d'eau à leur contact. Les parkings par leur nombre peuvent aussi influencer la nappe. Les 8 principaux parkings drainant la nappe représentent à eux seuls 1,5 millions de $\mathrm{m}^{3} / \mathrm{an}$; 




Fig. 3. - Carte hydrogéologique de DIFFRE, 1965 (tirée de DIFFRE, 1969).

Fig. 3. - Hydrogeological map. From DIFFRE, 1969.

- enfin le niveau des nappes baisse tout simplement parce qu'elles sont mal réalimentées. Les aménagements urbains ne laissent guère d'espaces verts pour que l'eau puisse s'infiltrer (dômes des Bois de Boulogne et Vincennes).

Une des nombreuses conséquences de ce phénomène a été la baisse de $12 \mathrm{~m}$ environ enregistrée entre 1854 et 1960 dans le quartier de l'Opéra. Les alluvions sont encore sèches sur une large zone en centre rive droite.

\section{MODIFICATIONS DU COMPORTEMENT DES NAPPES PHRÉATIQUES PENDANT LES ANNÉES 70-80}

Après cette chute des niveaux piézométriques jusqu'aux années 1965-70, un équilibre semblait devoir s'instaurer. Depuis 1975, quelques incidents indiquent une tendance à la remontée. Parmi les premières manifestations, on peut citer la condamnation de deux sous-sols d'un parking aux Champs Elysées, des venues d'eaux importantes Porte Maillot sur un ouvrage juste terminé, des altérations de matériaux comme au Pont de Neuilly.

Il est également à noter des incidents plus dommageables pour la stabilité des ouvrages en zone de gypses antéludiens. La baisse des niveaux des nap- pes en règle générale a modifié les écoulements dans les aquifères et les équilibres physico-chimiques. En particulier, une partie du gypse antéludien s'est trouvée en zone non saturée et s'est dissoute. Lors de la remontée, les nappes ont de nouveau saturé des horizons décomprimés, ont noyé des sous-sols et ont accéléré le déséquilibre des terrains. Une des solutions choisies par les propriétaires pour éviter d'ennoyer les ouvrages a été de pomper directement dans les nappes afin de les rabattre localement. Les effets ont été rapides : ces pompages ont accéléré les décompressions et déstabilisé les fondations des immeubles concernés.

A $95 \%$ ceci résulte d'une conjugaison de facteurs humains.

En premier lieu, le ralentissement des pompages industriels dans Paris qui résultent du départ de certaines entreprises et de l'application de la taxe parafiscale (1964) sur les prélèvements d'eau de nappe, peut expliquer une forte proportion de cette remontée.

Le débit total des prélèvements déclarés dans la nappe phréatique a chuté de $1510^{6} \mathrm{~m}^{3}$ en 1971 et $510^{6} \mathrm{~m}^{3}$ en 1982 à Paris (fig. 4).

La carte de COLIN (fig. 5) a été réalisée en superposant celle des hydroisohypses de 1982 en centre 



Fig. 5. - Superposition carte piézométrique. Carte des prélèvements (1982) en rive droite (tirée de COLIN, 1986).

Fig. 5. - Superposition of the piezometric map and of the map on the Right Bank, 1982. From COLIN, 1986 
rive droite à celle des débits déclarés en 1982. On obtient une forte corrélation entre le maximum des débits et le maximum de rabattement de la nappe phréatique.

Aux décentralisations industrielles s'ajoutent les techniques de recyclage d'eau (climatisation - réfrigération avec réinjections), la nette régression du nombre des grands travaux et de leur emprise sur la nappe, l'encombrement du sous-sol... Du fait de l'alimentation de la nappe par la Seine, le réaménagement de cette dernière peut avoir une influence non négligeable. Il en est de même de tous les réseaux d'eaux artificiels pas toujours très étanches tels les égouts anciens, les canaux, les lacs artificiels...

Enfin un facteur naturel entre en jeu : la pluviométrie. En effet la période 1978-1983 a été spécialement pluvieuse et les crues de 1982 et 1983 importantes.

\section{TENDANCES ACTUELLES AU VU DES RELÉVÉS DE L'INSPECTION GÉNÉRALE DES CARRIÈRES}

Depuis 1978, l'IGC suit l'évolution de quelques piézomètres (15 en 1978, 30 en 1981, 80 en 1989, 100 en 1990). Ce suivi a mis en évidence une remontée de la nappe très nette en centre rive droite (fig. 7). La nappe phréatique semble avoir atteint un pseudoéquilibre depuis 1983, jusqu'à de nouvelles modifications non prévisibles actuellement (nouveaux pompages, travaux souterrains, réalisation et étanchement de réseaux souterrains actuellement drainants ou futurs...).

D'autres constatations ont pu être faites aussi. Ainsi en rive gauche les nappes ont très peu évolué (fig. 6). A la Concorde en proximité de la Seine $(130 \mathrm{~m})$, on observe l'influence des crues de 1982 et 1983. On constate que la crue de 1982 a élevé de $0,8 \mathrm{~m}$ le niveau de la nappe et celle de 1983 de $0,5 \mathrm{~m}$. Etant donné l'importance de ces crues, on peut en conclure que le lit de la Seine doit être en partie colmaté.

De tous ces relevés piézométriques, deux types de synthèses sont faites à l'IGC :

- des cartes piézométriques sont réalisées soit à partir de modèle mathématique comme la carte de COLIN (1986), (fig. 8) dont il faut s'astreindre des effets de bord, soit à partir des relevés directement comme la

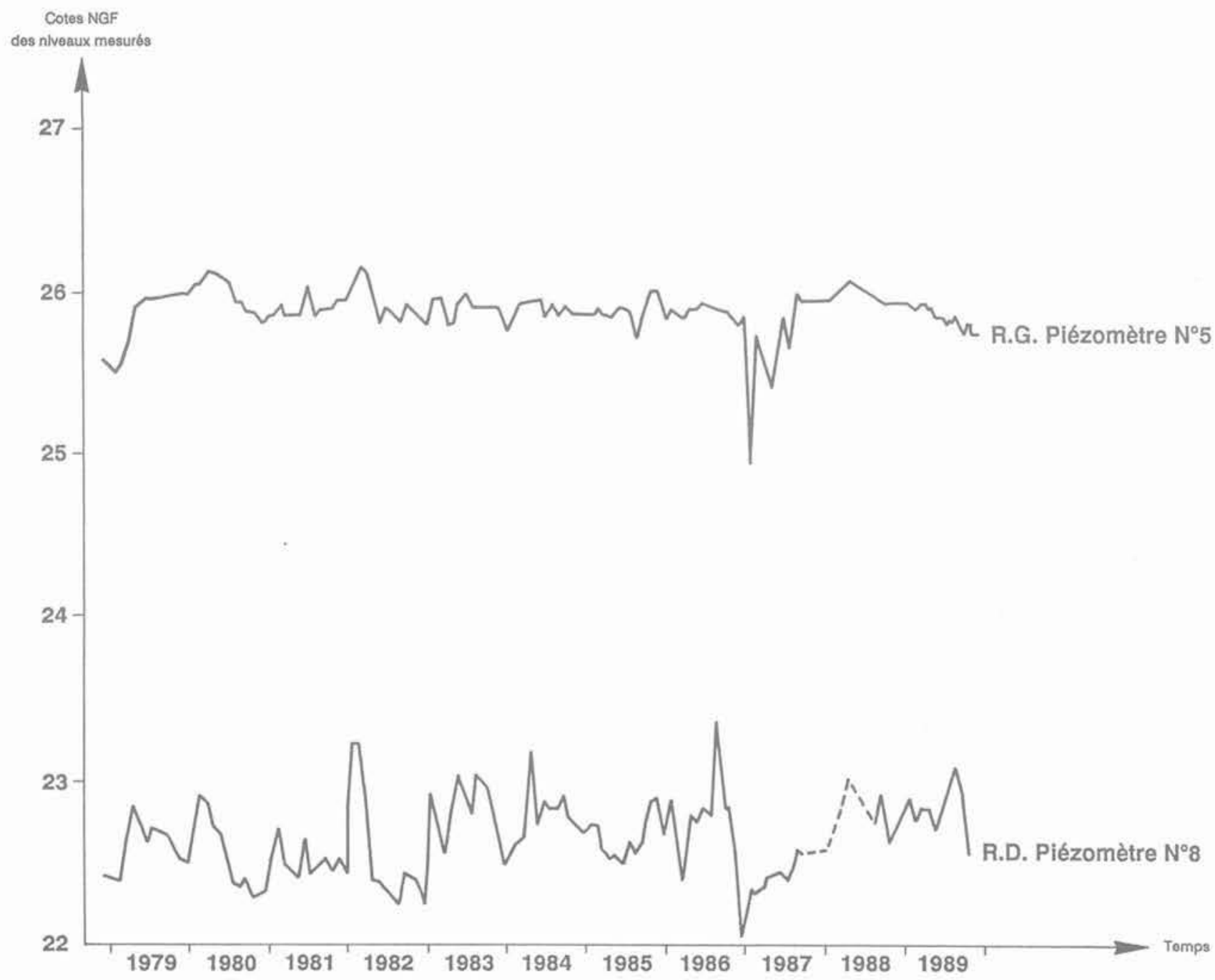

Fig. 6. - Evolution dans le temps du niveau piézométrique de 1978 à 1989 de piézomètres $n^{\circ} 5$ et $n^{\circ} 8$ du réseau IGC (doc. IGC). Fig. 6. - IGC network piezometer 5 and 8 level as a function of time. From IGC. 


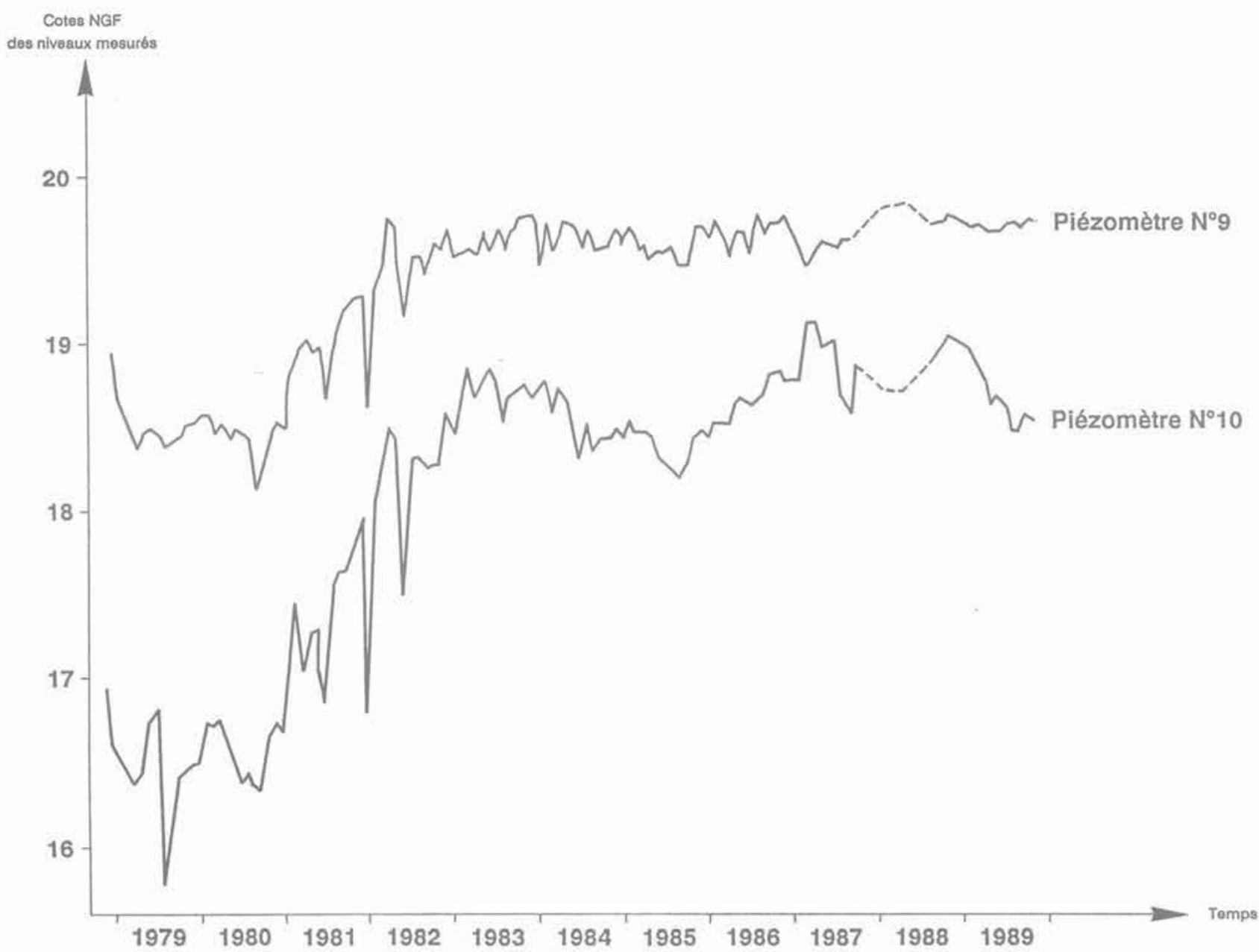

Fig. 7. - Evolution dans le temps du niveau piézométrique de 1978 à 1989 des piézomètres $n^{\circ} 9$ et $n^{\circ} 10$ du réseau IGC (doc. IGC).

Fig. 7. - IGC network piezometer 9 and 10 level as a function of time. From IGC.

carte d'octobre 1989 de PRUNIER-LEPARMENTIER (fig. 9) (il a été préféré une carte piézométrique de 1989 à une de 1990 en raison des baisses enregistrées pour les uns et d'une certaine anarchie incontrôlée pour les autres probablement due à des modifications de débit de pompage). Ce dernier type de cartes permet de mettre en évidence des zones d'anomalies. Actuellement trois zones d'anomalies ont été mises en évidence. La première se situe dans le 19 e arrondissement. Elle correspond à la zone du décret de 1977 concernant les poches de dissolution de gypse. Tout se passe comme si des « cheminées" mettaient la nappe phréatique (Eocène supérieur) en liaison avec la nappe sous-jacente (nappe lutétienne). Les niveaux mesurés indiquent un équilibre local entre les deux nappes. La deuxième (emplacement du fossé Charles V) et la troisième (fin de l'ancien rû de Ménilmontant) zones se situent toutes les deux dans le centre rive droite. L'anomalie est négative (niveau prévu - niveau réel). Géologiquement chacune se caractérise par une forte épaisseur de remblais et la troisième comporte en plus des alluvions modernes. On rencontre dans les deux cas de l'eau en base de ces remblais indépendamment de la nappe lutétienne. Ces zones seront probablement modifiées au niveau de l'ancien lit de la Seine en fonction des mesures réalisées ;

- un rapport synthétique (9) donnant une carte de risques a aussi été réalisée. Cette carte au 1/25000 de 1985 définit cinq zones à indices de risques différents en fonction du nombre de sous-sols réalisables hors d'eau, c'est-à-dire hors nappe et/ou circulations d'eau comme on peut en rencontrer dans les formations de pente. Ce document n'est consultable qu'à l'IGC.

L'IGC augmente régulièrement son parc de piézomètres, 90 ont été suivis mensuellement cette année, 20 viennent d'être forés. Ces mesures grossissent la banque de données piézométriques qui est en cours d'informatisation. Une enquête a également été réalisée afin de connaître les besoins et les souhaits des utilisateurs et de déterminer l'opportunité de créer un bulletin d'informations hydrogéologiques.

Enfin un projet ambitieux de banque de données commune avec le BRGM et l'Agence Financière de Bassin est à l'étude. Il a pour nom * GOEPARIF * et devrait permettre d'établir plus aisément des cartes hydroisohypses et des courbes d'évolution historique. 


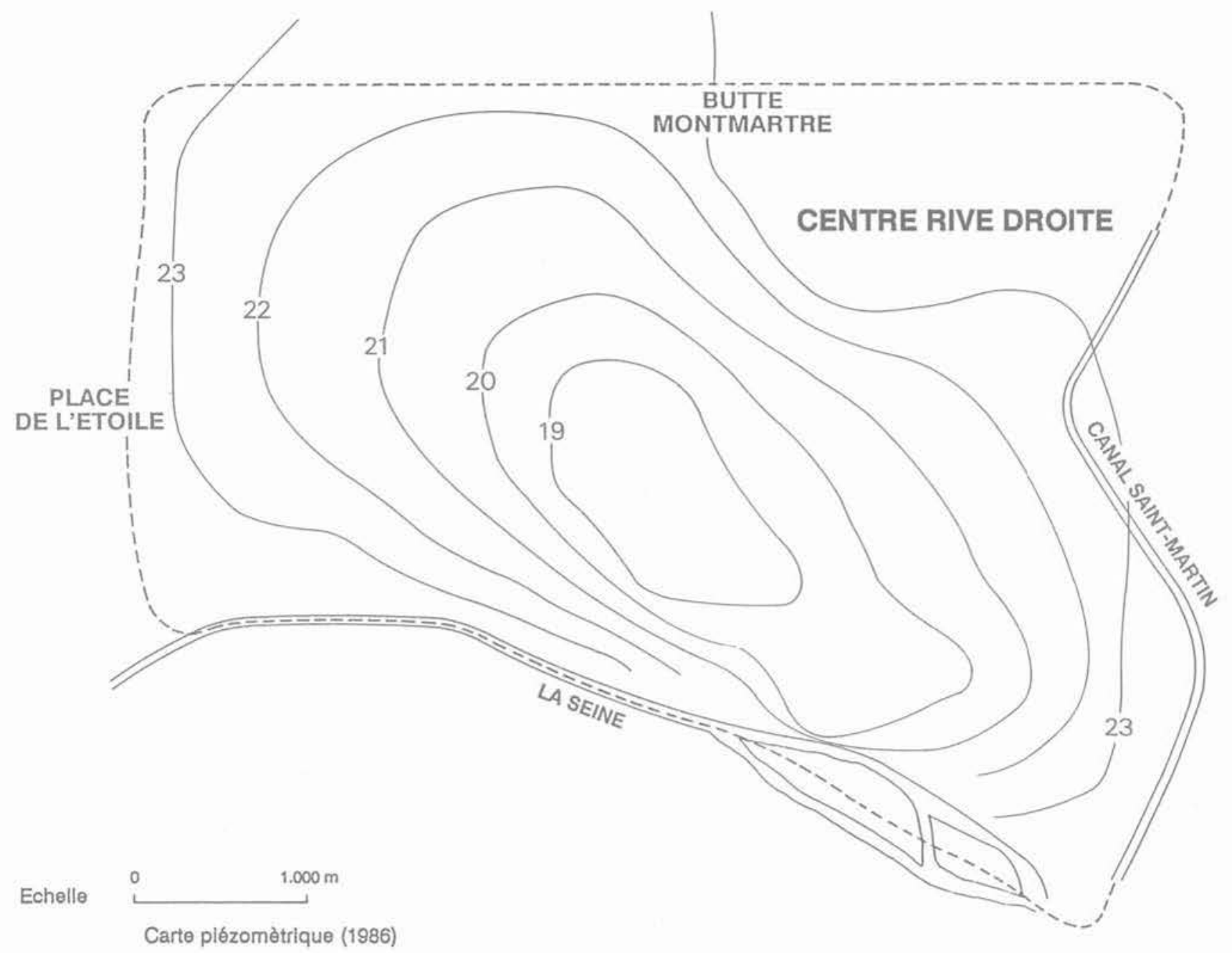

Fig. 8. - Carte piézométrique du Centre Rive Droite obtenue par simulation informatique (tirée de COLIN, 1986).

Fig. 8. - Informatic modelisation of the Right Bank Central part piezometric map. From COLIN, 1986.

30.- HYROISOHYPSES (NGF LALLEHAND)



tracée à partir des relevés de la dernière

semaine d'octobre 1989 des piézomètres de I'TGC faite par PRUNIER-LEPARMENTIER (1989),

Fig. 9. - Piezometric map in october 1989. From PRUNIER-LEPARMENTIER, 1989. 
Le but de toutes ces études et de ces moyens futurs et/ou actuels est de renseigner le public le plus justement possible, bien que ces renseignements ne soient qu'indicatifs et en aucune manière extrapolables. Certains projets, parfois même de simples forages mal rebouchés, peuvent en effet modifier temporairement voire définitivement les écoulements de nappe.

\section{BIBLIOGRAPHIE}

(1) BERGER G., MARQUET G. (1983), Etude de l'évolution piézométrique des nappes d'eau souterraines à Paris et en proche banlieue au cours des dix dernières années. Rapport BRGM 83 SGN 098 IDF, février 1983, 26 p.

(2) BERGERON G., DESHAYS H., POINTET T. (1983), Remontées des nappes d'eau souterraines, causes et effets. Doc. BRGM n 60,1983 , $100 \mathrm{p}$.

(3) COLIN P.L. (1986), Evolution des nappes peu profondes à Paris depuis un siècle. PFE, ENSMP, Paris, juillet 1986, 62 p.

(4) DELAFON H. (1981), Etude de la nappe phréatique à Paris. PFE, ENPC, Paris, 1981.
(5) DELESSE (1858), Carte hydraulique de Paris.

(6) DIFFRE P. (1969), Géologie dynamique. Hydrologie de Paris et de sa banlieue. Thèse de Doctorat $3^{e}$ cycle, Paris, 1969, 345 p.

(7) DIFFRE P. (1979), Désordres dus à la remontée du niveau des nappes peu profondes sous Paris. Doc. BRGM n 8, Lyon, mars 1979 , pp. 593-598.

(8) DIFFRE P., POMEROL C. (1979), Paris et ses environs. Guide géologique. Paris, Masson, $171 \mathrm{p}$.

(9) MARVY J. (1986), Les problèmes posés par la nappe phréatique à Paris. Rapport interne IGC, 1986, 60 p. + annexes.

(10) PRUNIER-LEPARMENTIER A.M. (1984), Contexte géologique et géotechnique de la ville de Paris. Problèmes hydrogéologiques. TFE ENSG Nancy et mémoire de DEA en géologie de l'ingénieur, 1984, 85 p + annexes.

(11) PRUNIER-LEPARMENTIER A.M. (1988), Les problèmes géologiques et géotechniques de la ville de Paris. Thèse de l'ENSMP en géologie de l'ingénieur, Paris, décembre 1988, 244 p. 